\title{
Functionally conserved effects of rapamycin exposure on zebrafish
}

\author{
CEREN SUCULARLI $^{1,2}$, HUMA SHEHWANA ${ }^{1}$, CEM KUSCU $^{1}$, \\ DILAY CIGLIDAG DUNGUL ${ }^{3}$, HILAL OZDAG ${ }^{3}$ and OZLEN KONU ${ }^{1}$ \\ ${ }^{1}$ Department of Molecular Biology and Genetics, Faculty of Science, Bilkent University, Ankara 06800; \\ ${ }^{2}$ Department of Bioinformatics, Institute of Health Sciences, Hacettepe University, Ankara 06100; \\ ${ }^{3}$ Biotechnology Institute, Ankara University, Ankara 06010, Turkey
}

Received August 10, 2015; Accepted January 22, 2016

DOI: $10.3892 / \mathrm{mmr} .2016 .5059$

\begin{abstract}
Mechanistic target of rapamycin (mTOR) is a conserved serine/threonine kinase important in cell proliferation, growth and protein translation. Rapamycin, a well-known anti-cancer agent and immunosuppressant drug, inhibits mTOR activity in different taxa including zebrafish. In the present study, the effect of rapamycin exposure on the transcriptome of a zebrafish fibroblast cell line, ZF4, was investigated. Microarray analysis demonstrated that rapamycin treatment modulated a large set of genes with varying functions including protein synthesis, assembly of mitochondrial and proteasomal machinery, cell cycle, metabolism and oxidative phosphorylation in ZF4 cells. A mild however, coordinated reduction in the expression of proteasomal and mitochondrial ribosomal subunits was detected, while the expression of numerous ribosomal subunits increased. Meta-analysis of heterogeneous mouse rapamycin microarray datasets enabled the comparison of zebrafish and mouse pathways modulated by rapamycin, using Kyoto Encyclopedia of Genes and Genomes and Gene Ontology pathway analysis. The analyses demonstrated a high degree of functional conservation between zebrafish and mice in response to rapamycin. In addition, rapamycin treatment resulted in a marked dose-dependent reduction in body size and pigmentation in zebrafish embryos. The present study is the first, to the best of our knowledge, to evaluate the conservation of rapamycin-modulated functional pathways between zebrafish and mice, in addition to the dose-dependent growth curves of zebrafish embryos upon rapamycin exposure.
\end{abstract}

\section{Introduction}

Rapamycin inhibits mechanistic target of rapamycin (mTOR), a conserved serine/threonine kinase that responds to growth

Correspondence to: Dr Ozlen Konu, Department of Molecular Biology and Genetics, Faculty of Science, Bilkent University, Ankara 06800, Turkey

E-mail: konu@fen.bilkent.edu.tr

Key words: meta-analysis, microarray, mouse, mTOR, rapamycin, real-time qPCR, zebrafish, ZF4 factors, nutrients and hypoxia (1). Rapamycin binds to the kinase domain of mTOR upon complexing with FK506 binding protein 12 (FKBP12) in TOR complex 1 (TORC1) (2) or through the phosphorylation of rictor, a necessary component of TORC2 (3). As a result, rapamycin inhibits growth in various cell types and thus is a promising anti-cancer agent (4).

The effect of rapamycin on mTOR function is highly conserved between species from yeast to humans $(5,6)$. In addition, microarray analyses testing the extent of global alterations in the transcriptome in yeast and in mammals contribute to the understanding of the mechanisms and pathways associated with the effect of rapamycin (7-12). In vivo studies indicate that rapamycin results in developmental delay in different organisms including Drosophila melanogaster, zebrafish and mice $(6,13,14)$. Previous studies using zebrafish have identified significant effects of rapamycin on autophagy (15), prevention of hepatic steatosis (16), heart development (17) and demonstrated the importance of zebrafish as a mitochondrial and ribosomal disease model $(18,19)$. Although the zebrafish is an emerging model in drug-screening and in vivo disease models (20), evolutionarily conserved effects of rapamycin on the zebrafish transcriptome in addition to the dose-dependency in embryonic/larval size and pigmentation have not previously been studied.

In the present study, the transcriptional response of zebrafish embryonic fibroblast cell line, ZF4, was characterized following exposure to $100 \mathrm{nM}$ rapamycin treatment for $48 \mathrm{~h}$. To the best of our knowledge, the current study is the first transcriptome analysis of zebrafish $\mathrm{ZF} 4$ cells in response to rapamycin treatment demonstrating significant alterations in the steady state mRNA levels of zebrafish cells. In addition, the present study confirmed the microarray results by reverse transcription-quantitative polymerase chain reaction (RT-qPCR) for a set of genes exhibiting large effect sizes. Significantly altered pathways indicated by Kyoto Encyclopedia of Genes and Genomes (KEGG) analysis included ribosomes, proteasomal machinery, oxidative phosphorylation and cell cycle. Notably, meta-analysis performed on microarray datasets of mouse cell lines treated with rapamycin revealed a high level of conservation between the mouse and zebrafish pathways modulated by rapamycin. Furthermore, statistical analysis of shared Gene Ontology (GO) terms between zebrafish and mice revealed a significant positive association. Additionally, the present study indicated that in vivo treatment of rapamycin 
resulted in a significant dose-dependent reduction in body size and in particular, pigmentation of zebrafish.

\section{Materials and methods}

Cell culture and rapamycin exposure. ZF4, a cell line derived from zebrafish embryonic fibroblast cells [CRL-2050 American Type Culture Collection (ATCC), Manassas, VA, USA], were cultured in Dulbecco's modified Eagle's medium/F-12 (1:1) (SH30023; GE Healthcare Life Sciences, Logan, UT, USA) containing 10\% fetal bovine serum (FBS; CH30160; GE Healthcare Life Sciences) with $1 \%$ streptomycin/penicillin (10,000 U/ml Penicillin, 10,000 $\mu \mathrm{g} / \mathrm{ml}$ Streptomycin; SV30010; GE Healthcare Life Sciences) at $28^{\circ} \mathrm{C}$. ZF4 cells were treated with rapamycin at a final concentration of $100 \mathrm{nM}$ (553210; EMD Millipore, Billerica, MA, USA) or dimethyl sufloxide (DMSO; $0.002 \%$ ) as control and collected at indicated time points. ZF4 cells previously were shown to be stable over multiple passages with no observable alterations in phenotype (21). ZF4 passage numbers $(\mathrm{P})$ used for different analyses following purchase from ATCC were as follows: P16, microarray analysis; P19, reverse transcription-quantitative polymerase chain reaction(RT-qPCR); P20, 3-(4,5-dimethylthiazol-2-yl)-2,5-diphenyltetrazolium bromide (MTT) assay; P21, propidium iodide (PI) analysis; P23, bromodeoxyuridine (BrdU) assay; and P19, cell death detection (CDD) analysis.

Cell viability and proliferation. $\mathrm{ZF} 4$ cells at a density of $2 \times 10^{4}$ were treated with $100 \mathrm{nM}$ rapamycin, DMSO or culture medium for $48 \mathrm{~h}$ in 96-well microplates prior to an MTT assay for cell viability (Vybrant MTT Cell Proliferation Assay kit, V-13154; Thermo Fisher Scientific, Inc., Waltham, MA, USA). The absorbance was read at $540 \mathrm{~nm}$ using a $\mu$ Quant $^{\mathrm{TM}}$ Microplate Spectrophotometer (Bio-Tek Instruments, Winooski, VT, USA)

ZF4 cells at a density of $5 \times 10^{5}$ were seeded in 6 -well plates in triplicate, and were treated with $100 \mathrm{nM}$ rapamycin or DMSO for $48 \mathrm{~h}$ for cell cycle analysis using PI $(50 \mu \mathrm{g} / \mathrm{ml})$ staining (22). For each sample, $1 \times 10^{4}$ cells were analyzed using a BD FACScalibur cell analyzer (BD Biosciences, San Jose, CA, USA).

ZF4 cells at a density of $5 \times 10^{5}$ cells were seeded in 6-well plates with $100 \mathrm{nM}$ rapamycin or DMSO for $48 \mathrm{~h}$ in triplicate, prior to the application of $30 \mu \mathrm{M}$ BrdU for $24 \mathrm{~h}$, to determine alterations in DNA synthesis. Subsequently, cells fixed with $70 \%$ ice cold ethanol were blocked with $10 \%$ FBS/phosphate-buffered saline (PBS) for $1 \mathrm{~h}$, and incubated with monoclonal mouse anti-BrdU antibody (1:500 in 2\% FBS in PBS; M0744; Dako, Glostrup, Denmark) for $1 \mathrm{~h}$ at room temperature, followed by goat anti-mouse secondary antibody conjugated to AlexaFluor 488 (1:750 in 2\% FBS; A11029; Thermo Fisher Scientific, Inc.) for $1 \mathrm{~h}$ at room temperature. The cells were counterstained with 4',6-diamidino-2-phenylindole (Roche Diagnostics, Basel, Switzerland) for $1 \mathrm{~min}$ and were analyzed in multiple fields using a Zeiss AX10 Imager A1 microscope (Carl Zeiss AG, Oberkochen, Germany).

The rate of apoptosis following rapamycin treatment was measured using a CDD enzyme-linked immunosorbent assay (ELISA) $^{\text {PLUS }}$ kit (Roche Diagnostics, GmbH, Mannheim, Germany) performed according to manufacturer's protocols following the treatment of $2 \times 10^{4} \mathrm{ZF} 4$ cells with $100 \mathrm{nM}$ rapamycin, DMSO or culture media for $48 \mathrm{~h}$ in 96 well plates, in duplicate. Absorbency was measured using an $\mu \mathrm{Quant}^{\mathrm{TM}}$ Microplate Spectrophotometer (Bio-Tek Instruments) at $405 \mathrm{~nm}$ using $490 \mathrm{~nm}$ as a reference.

Microarray experiment. A total of $5.5 \times 10^{6} \mathrm{ZF} 4$ cells, cultured in $\mathrm{T}-150$ flasks in triplicate, were treated with rapamycin at a final concentration of $100 \mathrm{nM}$ or DMSO for $48 \mathrm{~h}$ prior to collection. Total RNA was isolated using an SV Total RNA Isolation kit according to the manufacturer's instructions (Z3100, Promega Corporation, Madison, WI, USA). The quantity and quality of total RNA was assessed using an Agilent 2100 BioAnalyzer (Agilent Technologies, Inc., Santa Clara, CA, USA). All samples had RNA integrity number values greater than 8 . Total RNA converted to cDNA was in vitro transcribed in the presence of biotinylated nucleotides to produce biotin-labeled cRNA (antisense RNA, 3' IVT Express kit; Affymetrix, Santa Clara, CA, USA). Fragmented cRNA (Ambion; Thermo Fisher Scientific, Inc.) was hybridized to an Affymetrix GeneChip Zebrafish (Affymetrix, Inc., Santa Clara, CA, USA) according to the manufacturer's instructions. Following staining with a streptavidin-phycoerythrin conjugate (Invitrogen; Thermo Fisher Scientific, Inc.), the intensity emitted by the bound RNA was quantified using a GeneChip scanner (GeneChip Scanner 3000 7G; Affymetrix, Inc.). The Affymetric CEL files and normalized expression data were deposited into the Gene Expression Omnibus (GEO) database (GSE53086; http://www. ncbi.nlm.nih.gov/geo/).

Microarray data analysis. The Affymetrix GeneChip Zebrafish genome array contains $\sim 15,600$ probe sets. The quality control of the arrays and assessment of RNA integrity were performed in R software, version 2.10.1 (https://www.r-project.org) using affy (23) and affyPLM (24) packages. Raw microarray data were normalized using justRMA by Biometric Research Branch (BRB)-Array tools, version 4.2.1 (http://brb.nci.nih. gov/BRB-ArrayTools/download.html). The differentially expressed genes (fold change based on the geometric mean of intensities) under the rapamycin treatment were determined using the class comparison function of the BRB-array tools. The significance threshold was set at $\mathrm{P}<0.05$ for the univariate tests. Fisher's exact tests were then conducted with multiple test correction using Database for Annotation, Visualization and Integrated Discovery (DAVID; https://david.ncifcrf.gov/) 6.7 analysis $(25,26)$ to test the enrichment of the upregulated and downregulated probe sets indicated by KEGG and GO pathway analysis in zebrafish.

Meta-analysis of mouse rapamycin exposure microarray datasets. The GEO database was searched for microarray datasets performed using the Affymetrix Mouse Genome 430 2.0 Array in which mouse cell lines were treated with rapamycin. Samples that belong to a rapamycin treatment and associated control group were selected for further study. The CEL files were retrieved from three datasets (GSE21755, GSE19885 and GSE5332). Samples used from GSE21755 included rapamycin $(\mathrm{n}=2,20 \mathrm{nM}$ for $24 \mathrm{~h})$ and control ( $\mathrm{n}=3$, DMSO for $24 \mathrm{~h}$ ) treatments on two different tuberous sclerosis (Tsc) null mouse embryonic fibroblast lines (MEFs), Tsc1 ${ }^{-/-}$(p53 $3^{+/+}, 3 \mathrm{~T} 3$-immortalized) and $\mathrm{Tsc}^{-/-}\left(\mathrm{p} 53^{-/-}\right.$, derived from a littermate of the wild-type cell 
Table I. Primers and the primer efficiencies used for the microarray confirmations.

\begin{tabular}{llll}
\hline Gene name & \multicolumn{1}{c}{ Forward primer (5'-3') } & Reverse primer (5'-3') & Primer efficiency \\
\hline foxm 1 & GACACATGACCCTGAAGGAGA & AGAGTGAGACAGCGGTTTGC & 1.90 \\
pah & TGTGGGTATCGTGAGGACAA & GGAGCTGTGGCGAATGTACT & 1.75 \\
cyp 6 b 1 & CAACACGGGACAAGAGCTG & CCCATAAGAACCTTACGCACA & 1.92 \\
tagln2 & GCTGGTACAGTGGATCGTCA & TGGATCTTCTTCACAGGCTTT & 1.98 \\
bambia & CCTGCCTGTACGGAGCTAGA & CTGGATCAGCCTGACGATTT & 1.85 \\
$d k k 1 b$ & TTTGCATGACCTCACACACC & CGGAACCACACCTCTTTAGC & 1.91 \\
wifl & CGCTATTAAAGTCGGTTCAGG & TTGCACTGGAGACAGACACC & 1.86 \\
$m m p 9$ & CAGACTCTGCGTTCTTTGGA & GGATGGTCACCTCAAATGCT & 2.00 \\
$b 2 m$ & CAAGACATTCGACGGAGACC & TGCTTTCCCGAAAGAGATCA & 1.95 \\
\hline
\end{tabular}

foxm1, forkhead box M1; pah, phenylalanine hydroxylase; cyp26b1, cytochrome P450, family 26, subfamily b, polypeptide 1; tagln2, transgelin 2; ddc, dopa decarboxylase; bambia, BMP and activin membrane-bound inhibitor (Xenopus laevis) homolog a; dkk1b, dickkopf 1b; wif1, wnt inhibitory factor $1 ; m m p 9$, matrix metalloproteinase $9 ; b 2 m$, beta-2-microglobulin.

Table II. Kyoto Encyclopedia of Genes and Genomes pathway results from the Database for Annotation, Visualization and Integrated Discovery for upregulated and downregulated genes in zebrafish (Benjamini <0.05) and respective results from mice meta-analysis for rapamycin treatment.

\begin{tabular}{|c|c|c|c|c|c|c|}
\hline \multirow[b]{2}{*}{ Genes } & \multicolumn{3}{|c|}{ Zebrafish microarray } & \multicolumn{3}{|c|}{ Mouse meta-analysis } \\
\hline & Count & P-value & Benjamini & Count & P-value & Benjamini \\
\hline \multicolumn{7}{|l|}{ Upregulated genes } \\
\hline Ribosome & 15 & $1.5 \times 10^{-06}$ & $1.3 \times 10^{-04}$ & 36 & $5.8 \times 10^{-18}$ & $9.3 \times 10^{-16}$ \\
\hline \multicolumn{7}{|l|}{ Downregulated genes } \\
\hline Oxidative phosphorylation & 42 & $1.9 \times 10^{-15}$ & $2.3 \times 10^{-13}$ & 25 & $4.2 \times 10^{-03}$ & $3.7 \times 10^{-02}$ \\
\hline Cell cycle & 32 & $4.9 \times 10^{-08}$ & $3.0 \times 10^{-06}$ & 27 & $6.9 \times 10^{-04}$ & $7.8 \times 10^{-03}$ \\
\hline Proteasome & 16 & $1.5 \times 10^{-05}$ & $6.1 \times 10^{-04}$ & 27 & $3.5 \times 10^{-14}$ & $3.0 \times 10^{-12}$ \\
\hline Valine, leucine and isoleucine degradation & 13 & $7.6 \times 10^{-05}$ & $2.3 \times 10^{-03}$ & 6 & $5.4 \times 10^{-01}$ & $8.2 \times 10^{-01}$ \\
\hline Glutathione metabolism & 11 & $6.2 \times 10^{-04}$ & $1.5 \times 10^{-02}$ & 11 & $4.2 \times 10^{-02}$ & $1.9 \times 10^{-01}$ \\
\hline Progesterone-mediated oocyte maturation & 20 & $7.3 \times 10^{-04}$ & $1.5 \times 10^{-02}$ & 9 & $6.9 \times 10^{-01}$ & $8.9 \times 10^{-01}$ \\
\hline Arginine and proline metabolism & 14 & $8.4 \times 10^{-04}$ & $1.5 \times 10^{-02}$ & 10 & $1.0 \times 10^{-01}$ & $3.5 \times 10^{-01}$ \\
\hline Glycolysis/gluconeogenesis & 14 & $1.2 \times 10^{-03}$ & $1.8 \times 10^{-02}$ & 19 & $1.6 \times 10^{-04}$ & $2.5 \times 10^{-03}$ \\
\hline N-glycan biosynthesis & 11 & $2.6 \times 10^{-03}$ & $3.5 \times 10^{-02}$ & 6 & $5.4 \times 10^{-01}$ & $8.2 \times 10^{-01}$ \\
\hline Pyruvate metabolism & 10 & $3.1 \times 10^{-03}$ & $3.7 \times 10^{-02}$ & 11 & $8.6 \times 10^{-03}$ & $6.1 \times 10^{-02}$ \\
\hline Oocyte meiosis & 21 & $3.9 \times 10^{-03}$ & $4.2 \times 10^{-02}$ & 15 & $3.3 \times 10^{-01}$ & $6.7 \times 10^{-01}$ \\
\hline Fatty acid metabolism & 9 & $4.5 \times 10^{-03}$ & $4.5 \times 10^{-02}$ & 3 & $9.6 \times 10^{-01}$ & $9.9 \times 10^{-01}$ \\
\hline Tricarboxylic acid cycle & 9 & $4.5 \times 10^{-03}$ & $4.5 \times 10^{-02}$ & 7 & $1.0 \times 10^{-01}$ & $3.5 \times 10^{-01}$ \\
\hline
\end{tabular}

Benjamini, Benjamini-Hochberg corrected P-value.

line) (11). From GSE19885, rapamycin (100 nM for $24 \mathrm{~h}$; $\mathrm{n}=3$ ) and control (DMSO for $24 \mathrm{~h} ; \mathrm{n}=3$ ) treated samples from a rapamycin sensitive mouse brain tumor cell line, $\mathrm{BC} 3 \mathrm{H} 1$, were used (12). From GSE5332, TSC2 $2^{-/}$MEFs treated with $20 \mathrm{nM}$ rapamycin or DMSO for $14 \mathrm{~h}(\mathrm{n}=6)(10)$ were included in the meta-analysis. The quality control analysis of the CEL files was performed using R packages affyQCReport (https://www. bioconductor.org/packages/release/bioc/html/affyQCReport. html) (23) and affyPLM (https://www.bioconductor. org/packages/release/bioc/html/affyPLM.html) (24). One sample (GSM497116) from GSE19885 did not pass the quality control check thus was excluded from further analysis. CEL files normalized using robust multichip average (23) were subjected to meta-analysis using MetaDE Bioconductor package (https://cran.r-project.org/web/packages/MetaDE/index. html) (27). Normalized expression data along with control and treatment sample labels for each GSE dataset were given as input to meta-analysis function for calculation of z-scores, $\mathrm{P}$-values and the false-discovery rate (FDR) using a random effects model (1,000 permutations; seed value set to 123). 
Table III. Counts for the significant $(\mathrm{P}<0.05)$ shared GO terms in mice and zebrafish.

\begin{tabular}{|c|c|c|c|c|}
\hline \multirow[b]{3}{*}{ Zebrafish } & \multicolumn{4}{|c|}{ Mice } \\
\hline & \multicolumn{2}{|c|}{ All shared terms } & \multicolumn{2}{|c|}{$\begin{array}{l}\text { Ambiguous } \\
\text { terms removed }\end{array}$} \\
\hline & Down & Up & Down & Up \\
\hline \multicolumn{5}{|l|}{ GO-BP } \\
\hline Down & 54 & 13 & 43 & 2 \\
\hline Up & 3 & 18 & 1 & 16 \\
\hline \multicolumn{5}{|l|}{ GO-CC } \\
\hline Down & 23 & 8 & 17 & 3 \\
\hline Up & 12 & 8 & 3 & 0 \\
\hline \multicolumn{5}{|l|}{ GO-MF } \\
\hline Down & 18 & 2 & 17 & 1 \\
\hline $\mathrm{Up}$ & 5 & 6 & 4 & 5 \\
\hline
\end{tabular}

$\mathrm{GO}$, gene ontology; BP, biological process; CC, cellular component; MF, molecular function.

Filtered probes with FDR $<0.005$ (corresponding P-value: $\left.5.7 \times 10^{-4}\right)$ were used for further DAVID analysis. Meta-analysis was conducted in R software, version 3.1.2. Significant KEGG and GO pathways were extracted using DAVID for up- and downregulated mouse probe sets separately and then associated with the zebrafish functional pathway results.

$R T-q P C R$ validation experiments. Results from the microarray data were confirmed for selected significantly upregulated or downregulated genes by RT-qPCR analysis. An independent set of rapamycin treatment experiments was performed in which $5 \times 10^{6} \mathrm{ZF} 4$ cells were treated with $100 \mathrm{nM}$ rapamycin or DMSO in triplicate for 6 or $48 \mathrm{~h}$ prior to collection. For each sample, $1.2 \mu \mathrm{g}$ RNA, reverse transcribed into cDNA according to the manufacturer's instructions (K1622; Fermentas; Thermo Fisher Scientific, Inc.), was amplified with sequence specific primers designed using Primer3 (Table I; http://frodo.wi.mit. edu/primer3/). qPCR reactions were performed using an iCycler (Bio-Rad Laboratories, Inc., Hercules, CA, USA) for each sample in duplicate, using DyNAmo HS SYBR Green qPCR Kit (F-410 L; Thermo Fisher Scientific, Inc.). The running parameters were as follows: 1 cycle at $95^{\circ} \mathrm{C}$ for $10 \mathrm{~min}$, followed by $45 \mathrm{cycles}$ of $95^{\circ} \mathrm{C}$ for $30 \mathrm{sec}, 60^{\circ} \mathrm{C}$ for $30 \mathrm{sec}, 72^{\circ} \mathrm{C}$ for $30 \mathrm{sec}$, and finally $72^{\circ} \mathrm{C}$ for $10 \mathrm{~min} . b 2 \mathrm{~m}$ was used as the reference gene (28). The 10-fold serial dilutions were used to identify the primer efficiencies (E) (Table I). A modified $2^{-\Delta \Delta C q}$ method (29) was applied to calculate fold expression change of selected genes: $\left(\mathrm{E}_{\text {target }}\right)^{\Delta \mathrm{C} \text { Target (control-sample) }} /\left(\mathrm{E}_{\text {ref }}\right)^{\Delta \mathrm{C} \text { tReference (control-sample) }}$. The fold changes values were $\log _{2}$ transformed prior to plotting and performing statistical analyses.

Rapamycin treatment of zebrafish embryos. Shield stage zebrafish embryos (EMBL Heidelberg, Heidelberg, Germany) were placed into 96-well plates; each well contained 3 embryos. Final concentrations of 2, 10 and $20 \mu \mathrm{M}$ rapamycin (R0395;
Sigma-Aldrich, St. Louis, MO, USA) or equivalent DMSO concentrations were added to the embryo medium. Zebrafish embryos were collected at 1-5 days post fertilization (dpf) for further examination. The study was approved by the Bilkent University Local Animal Ethics Committee (No. 2003/5; Bilkent University, Ankara, Turkey); and in vivo zebrafish rapamycin exposure experiments were performed at European Molecular Biology Laboratory (Heidelberg, Germany) in compliance with local animal care regulations.

Statistical analysis. Minitab software, version 13.20 (Minitab Inc., State College, PA, USA) was used to analyze the significance of the RT-qPCR, MTT, BrdU, CDD and PI staining and in vivo results. The significance between groups was detected with Fisher's pairwise comparisons following a one-way analysis of variance (ANOVA). RT-qPCR results from 6 and $48 \mathrm{~h}$ rapamycin or equivalent of DMSO treatments were analyzed with either one-way or two-way ANOVA as necessary. A general linear model was applied to detect the significance of dose- and time-dependent rapamycin treatment on body length in vivo in zebrafish embryos. The graphs were generated using GraphPad Prism software, version 5.0 (GraphPad Software, Inc., San Diego, CA, USA). P $<0.05$ was considered to indicate a statistically significant difference. The Pearson correlation coefficient and associated P-value were calculated using the cor.test function in R. Functional analyses were performed using DAVID version 6.7. For DAVID based pathways and GO analyses, P-values corrected using the Benjamini Hochberg method were reported; Benjamini $<0.05$ was considered to indicate a statistically significant difference. Zebrafish and mouse GO term lists [biological process (BP); cellular compartment (CC); molecular function (MF)] were matched with each other; and shared terms having $\mathrm{P} \leq 0.05$ were tested for a significant positive association using odds ratios (OR, based on the conditioned maximum likelihood estimate) calculated using two-sided Fisher's exact test from the exact $2 \times 2$ package in R software, version 3.1.2 (30,31).

\section{Results}

Rapamycin reduces basal apoptosis with no detectable alteration in cell viability or proliferation in ZF4 cells. The results indicated that $100 \mathrm{nM}$ rapamycin did not have significant effects on cytotoxicity or DNA synthesis in ZF4 cells (one-way ANOVA; MTT, $\mathrm{P}=0.47$; $\mathrm{BrdU}, \mathrm{P}=0.141$; data not shown). In addition, cell fractions in $G_{0} / G_{1}, S$ and $G_{2} / M$ phases or in subG1 phase did not differ between the rapamycin and DMSO treated groups (one-way ANOVA for subG1 phase; $\mathrm{P}=0.394$; data not shown). Notably, however, the ELISA-based CDD analysis showed that rapamycin significantly reduced the basal apoptosis in ZF4 cells (one-way ANOVA; $\mathrm{P}=0.01$; Fig. 1).

Rapamycin affects steady state $m R N A$ expression in zebrafish ZF4 cells. The differentially expressed genes between rapamycin and DMSO control groups were obtained using the class comparison function of the BRB array tools $(\mathrm{P}<0.05)$. This model is preferable for data with small number of samples since it uses a random variance model for univariate tests to compute a P-value for each probe set together with an FDR value (32). A total of 2,186 probe sets 
Table IV. Top five shared significant (Benjamini <0.05) GO-BP term results from Database for Annotation, Visualization and Integrated Discovery categories for the upregulated and downregulated genes following rapamycin treatment.

\begin{tabular}{|c|c|c|c|c|c|c|}
\hline \multirow[b]{2}{*}{ GO-BP terms } & \multicolumn{3}{|c|}{ Zebrafish microarray } & \multicolumn{3}{|c|}{ Mouse meta-analysis } \\
\hline & Count & P-value & Benjamini & Count & P-value & Benjamini \\
\hline \multicolumn{7}{|l|}{ Upregulated genes } \\
\hline GO:0006412; translation & 25 & $6.9 \times 10^{-05}$ & $1.1 \times 10^{-02}$ & 52 & $1.5 \times 10^{-07}$ & $6.1 \times 10^{-05}$ \\
\hline GO:0048598; embryonic morphogenesis & 23 & $6.3 \times 10^{-05}$ & $1.3 \times 10^{-02}$ & 48 & $1.0 \times 10^{-04}$ & $7.4 \times 10^{-03}$ \\
\hline GO:0048568; embryonic organ development & 17 & $1.8 \times 10^{-04}$ & $2.0 \times 10^{-02}$ & 34 & $4.8 \times 10^{-04}$ & $2.5 \times 10^{-02}$ \\
\hline $\begin{array}{l}\text { GO:0009792; embryonic development ending } \\
\text { in birth or egg hatching }\end{array}$ & 16 & $1.8 \times 10^{-04}$ & $2.3 \times 10^{-02}$ & 55 & $7.2 \times 10^{-05}$ & $5.6 \times 10^{-03}$ \\
\hline GO:0043009; chordate embryonic development & 16 & $1.8 \times 10^{-04}$ & $2.3 \times 10^{-02}$ & 54 & $1.1 \times 10^{-04}$ & $7.5 \times 10^{-03}$ \\
\hline \multicolumn{7}{|l|}{ Downregulated genes } \\
\hline GO:0006259; DNA metabolic process & 35 & $5.0 \times 10^{-07}$ & $1.3 \times 10^{-04}$ & 79 & $4.7 \times 10^{-08}$ & $4.8 \times 10^{-06}$ \\
\hline GO:0007049; cell cycle & 31 & $3.9 \times 10^{-07}$ & $1.4 \times 10^{-04}$ & 96 & $7.1 \times 10^{-06}$ & $3.8 \times 10^{-04}$ \\
\hline $\begin{array}{l}\text { GO:0006091; generation of precursor metabolites } \\
\text { and energy }\end{array}$ & 28 & $8.0 \times 10^{-07}$ & $1.4 \times 10^{-04}$ & 62 & $1.6 \times 10^{-10}$ & $2.7 \times 10^{-08}$ \\
\hline GO:0005996; monosaccharide metabolic process & 19 & $9.8 \times 10^{-06}$ & $7.2 \times 10^{-04}$ & 41 & $4.5 \times 10^{-06}$ & $2.5 \times 10^{-04}$ \\
\hline GO:0019318; hexose metabolic process & 18 & $2.0 \times 10^{-05}$ & $1.2 \times 10^{-03}$ & 39 & $1.3 \times 10^{-06}$ & $8.0 \times 10^{-05}$ \\
\hline
\end{tabular}

GO-BP, gene ontology-biological process; Benjamini, Benjamini-Hochberg corrected P-value.

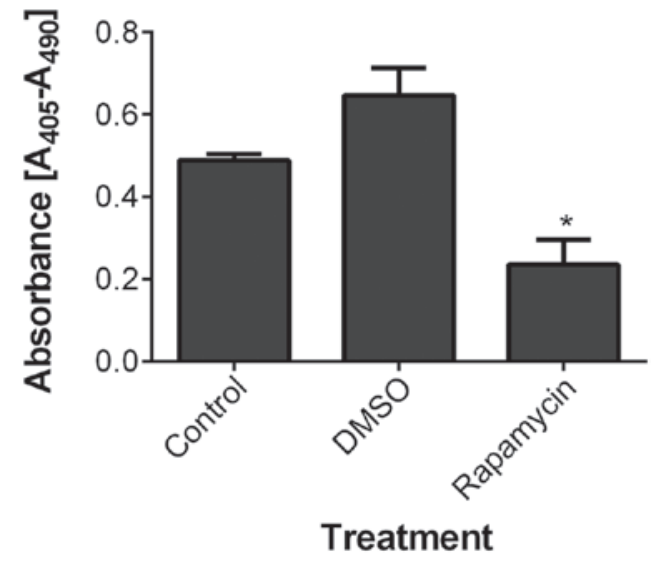

Figure 1. Cell death detection assay of rapamycin treated ZF4 cells. One-way analysis of variance with Fisher's multiple comparisons were performed to test the significance $(*)$ of rapamycin treatment from the control $(\mathrm{P}=0.017)$ and DMSO (P=0.004). DMSO, dimethyl sufloxide.

(904 upregulated and 1,282 downregulated) were detected as significantly modulated between the rapamycin and DMSO groups at $\mathrm{P}<0.05$.

$R T-q P C R$ validation and microarray results are correlated. To validate the microarray results, RT-qPCR was used to test whether rapamycin treated $\mathrm{ZF} 4$ cells exhibited time-dependent alterations in gene expression (at 6 and $48 \mathrm{~h}$ ). A total of nine genes (seven upregulated and two downregulated) were selected that exhibited high fold-change differences, low P-values and FDRs. Of the nine selected genes, six showed statistically significant alterations in time or treatment or both $(\mathrm{P}<0.05$; Fig. 2). These genes included dopa decarboxylase (ddc), cytochrome $\mathrm{P} 450$, family 26 , subfamily b, polypeptide 1 (cyp26b1), dickkopf 1b (dkklb), wnt inhibitory factor 1 (wifl), forkhead box M1 (foxml) and phenylalanine hydroxylase (pah) (Fig. 2). Overall, the $\log _{2}$ fold changes of all the selected genes at $48 \mathrm{~h}$ of rapamycin exposure were highly correlated between the microarray and RT-qPCR data (Pearson correlation coefficient, $r=0.79, \mathrm{P}=0.011$; Fig. 3).

KEGG pathway and GO enrichment analyses reveal high conservation between zebrafish and mouse in the response to rapamycin. According to KEGG pathway analysis in zebrafish, genes coordinately upregulated in response to rapamycin treatment were largely the components of small and large nuclear ribosomes, while those that were downregulated were enriched for proteasomal complexes, oxidative phosphorylation, the cell cycle, glycolysis/gluconeogenesis and certain metabolic pathways (Table II). Meta-analysis of the mouse datasets identified 5,019 consistently however, differentially expressed probe sets (FDR <0.005) among which 2,776 were downregulated and 2,243 were upregulated. KEGG pathway comparisons between zebrafish and mice revealed that ribosome was the most significantly upregulated pathway term, while several pathways downregulated in zebrafish were also downregulated in mice (Table II). Similarly, the shared GO terms between the zebrafish and mouse rapamycin datasets were identified (Table III). Commonly upregulated GO terms for CC included ribosomal and ribonucleoprotein complexes, while proteasome complexes, endoplasmic reticulum and mitochondrion terms were among the commonly downregulated CC terms (data not shown). However, Fisher's exact test for GO-CC did not indicate a significant positive association for the significant $(\mathrm{P} \leq 0.05)$ shared terms from mouse and zebrafish [OR, 1.89; (95\% CI, 0.53-6.52); $\mathrm{P}=0.36$; Table III]. Conversely, for GO terms of MF, an overall significant positive association was found between zebrafish and mice (OR, 9.79; (95\% CI, 1.58-86.01); $\mathrm{P}=1.2 \times 10^{-2}$; 
A

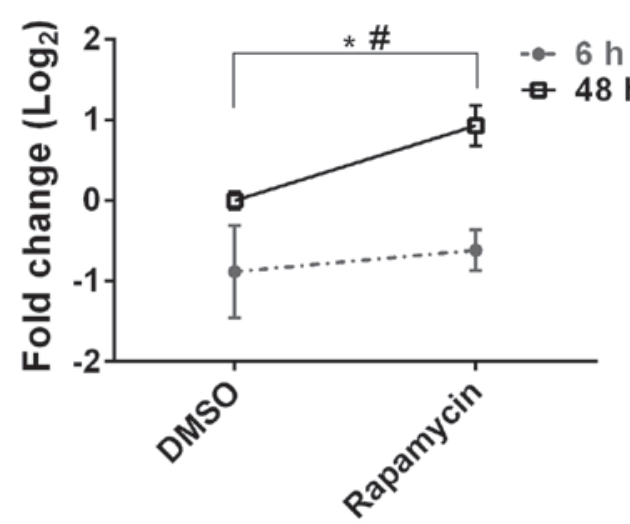

C

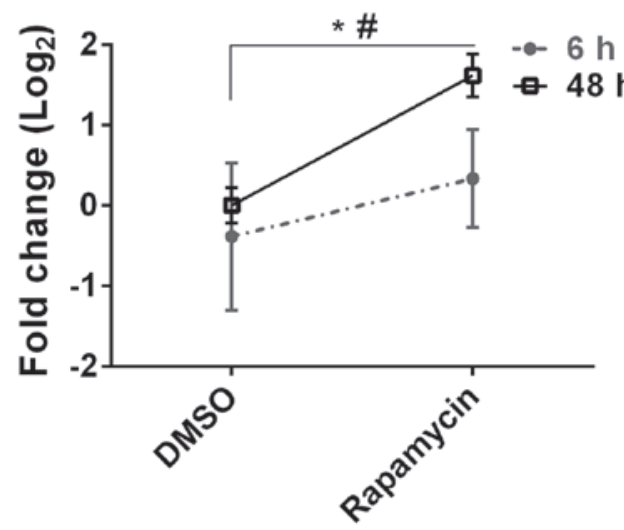

$\mathbf{E}$

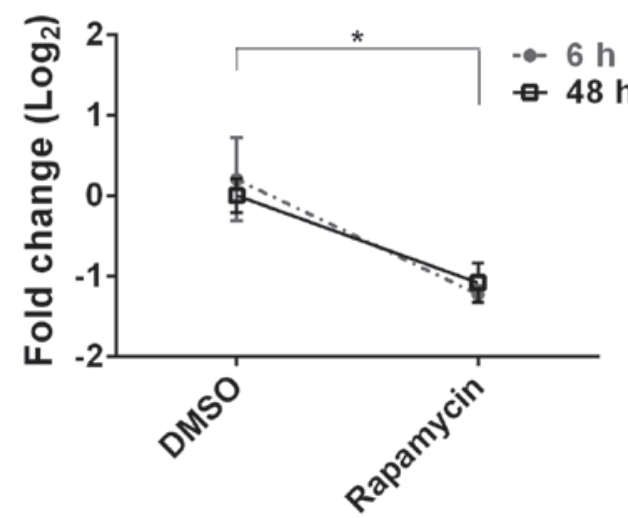

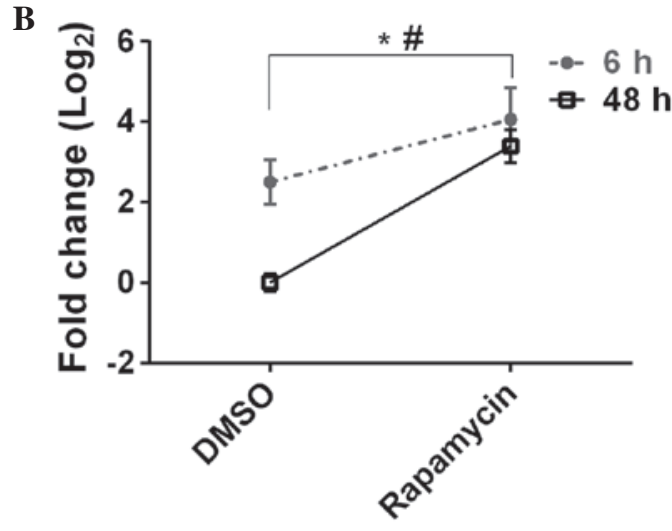

D

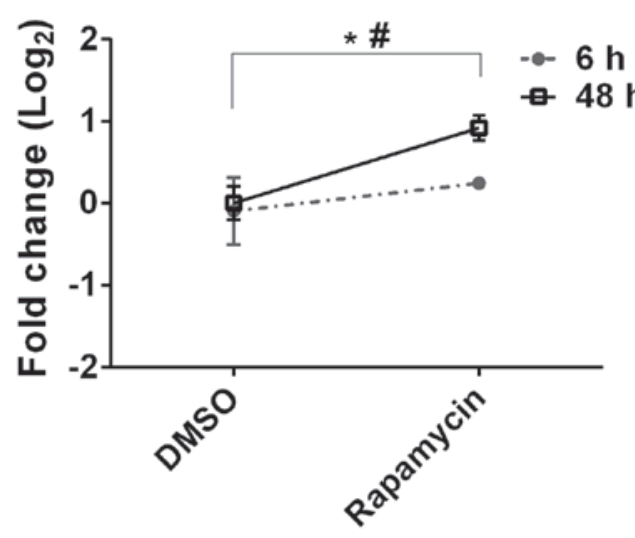

F

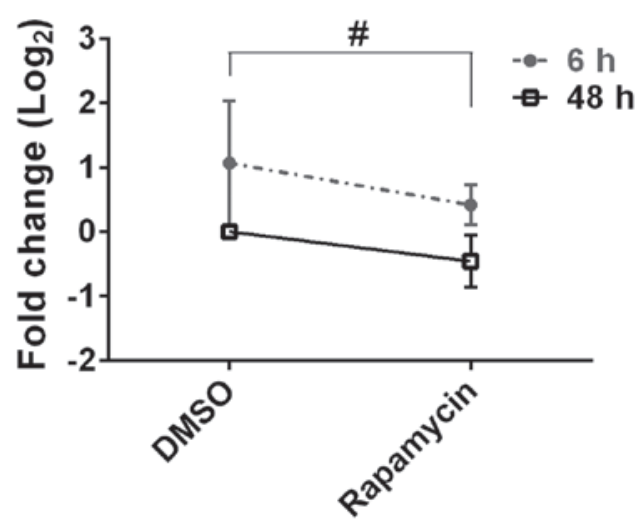

Figure 2. Genes showing significant modulations by microarray and RT-qPCR analyses. The significantly upregulated genes selected for RT-qPCR confirmation were (A) $d d c$, (B) $d k k 1 b$; (C) cyp26b1 and (D) wifl. Two-way ANOVA, $d d c, \mathrm{P}_{\text {treatment }}=0.022, \mathrm{P}_{\text {time }} \leq 0.001 ; d k k 1 b, \mathrm{P}_{\text {treatment }} \leq 0.001, \mathrm{P}_{\text {time }}=0.004 ;$ cyp26b1, $\mathrm{P}_{\text {treatment }}=0.008, \mathrm{P}_{\text {time }}=0.039 ;$ wif $1, \mathrm{P}_{\text {treatment }}=0.004, \mathrm{P}_{\text {time }}=0.039$. The significantly downregulated genes by rapamycin in RT-qPCR analysis were (E) pah and (F) foxml. Two-way ANOVA, pah, $\mathrm{P}_{\text {treatment }} \leq 0.001, \mathrm{P}_{\text {time }}=0.884$; foxml, $\mathrm{P}_{\text {treatment }}=0.098, \mathrm{P}_{\text {time }}=0.010 .{ }^{*} \mathrm{P}<0.05$ DMSO vs. rapamycin; ${ }^{*} \mathrm{P}<0.056 \mathrm{~h}$ vs. $48 \mathrm{~h}$. RT-qPCR, reverse transcription-quantitative polymerase chain reaction; ANOVA, analysis of variance; $d d c$, dopa decarboxylase; $d k k 1 b$, dickkopf $1 \mathrm{~b}$; cyp26b1, cytochrome P450, family 26, subfamily b, polypeptide 1; wifl, wnt inhibitory factor 1; pah, phenylalanine hydroxylase; foxm1, forkhead box M1; DMSO, dimethyl sulfoxide.

Table III). Several GO-MF categories, including DNA binding, structural constituent of ribosomes, threonine-type endopeptidase and peptidase activity, and cofactor binding, were common between zebrafish and mice. Similarly, shared zebrafish and mouse GO-BP terms were significantly and positively associated with each other [OR, 23.68 ; $(95 \% \mathrm{CI}$, 5.84-107.61); $\mathrm{P}=6.3 \times 10^{-8}$; Table III]. Upregulated GO-BP terms primarily included embryonic processes such as embryonic morphogenesis and embryonic organ development, while the top downregulated GO-BP terms were enriched in DNA metabolic process and the cell cycle, as in the KEGG pathway analysis (Table IV).
Some of the generalized GO terms, such as translation and cell cycle, were significant in both up and downregulated categories, particularly in mice. Following the removal of such ambiguous (both up and downregulated) terms, the P-values of the Fisher's test results for BP [OR, 253.65; (95\% CI, 25.08-7219.69); $\left.\mathrm{P}=9.1 \times 10^{-12}\right]$ and MF [OR, 18.22; (95\% CI, 1.52-521.42); $\mathrm{P}=7.9 \times 10^{-03}$ ] categories remained significant. However the Fisher's test for GO-CC remained insignificant.

The Fisher's tests for GO term association between zebrafish and mice were repeated for different FDR values of mouse meta-analysis (i.e. FDR $<0.05, \mathrm{FDR}<0.01$ and the top ranked 2,186 probes) to test whether the observed associations 


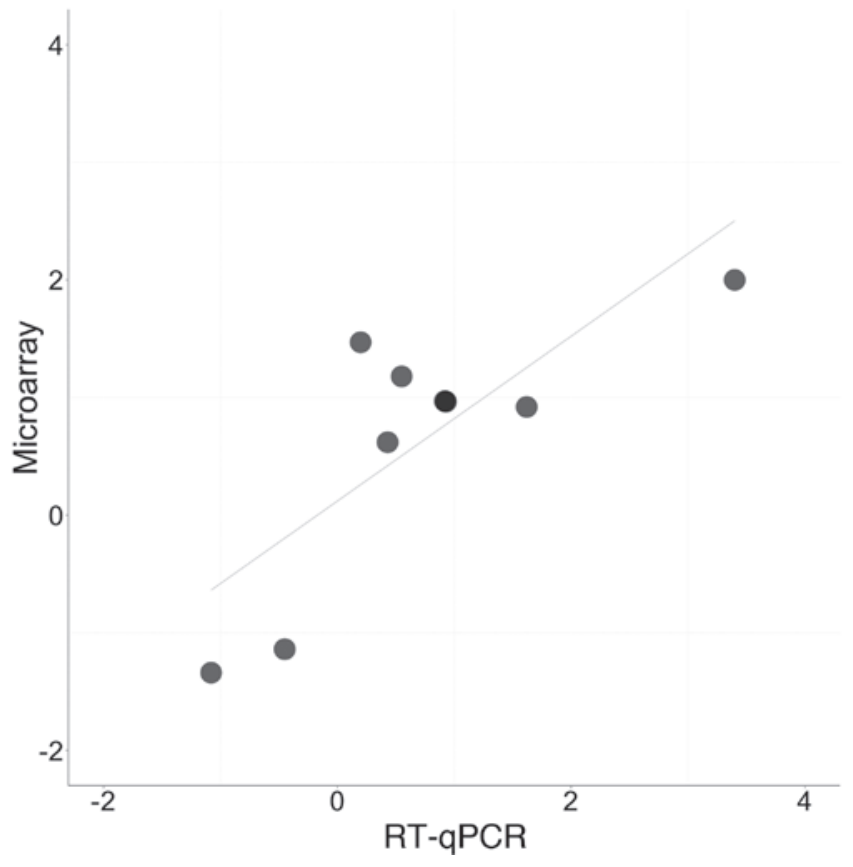

Figure 3. Pearson correlation plot for the microarray and RT-qPCR fold changes $\left(\log _{2}\right)$ for the selected upregulated and downregulated genes. RT-qPCR, reverse transcription-polymerase chain reaction.

at $\mathrm{FDR}<0.005$ were robust. Fisher's tests using probe sets specified at each FDR cut-off revealed that GO-BP (all terms) were significantly positively associated between zebrafish and mice regardless of the FDR values selected (FDR $<0.05$, $\mathrm{P}=3.1 \times 10^{-14}$; FDR $<0.01, \mathrm{P}=1.2 \times 10^{-09}$; top 2,186 ranked probes, $\left.\mathrm{P}=3.4 \times 10^{-05}\right)$. A similar result was observed for $\mathrm{GO}-\mathrm{MF}$ at FDR of $0.01\left(\mathrm{P}=7.8 \times 10^{-03}\right)$ and top 2,186 ranked probes $\left(\mathrm{P}=3.0 \times 10^{-02}\right)$, however, not for $\mathrm{FDR}<0.05(\mathrm{P}=0.12)$. Consistent with FDR value of 0.005 , the Fisher's test for GO-CC was not significant at any other selected value.

Effects of rapamycin on body size and pigmentation are dose-dependent. Rapamycin has been shown to limit growth in multiple organisms, however, the dose- and time-dependency of such effects have not been well assessed. Therefore, the effects of rapamycin on the development of zebrafish embryos (TLF) treated with increasing doses of rapamycin were assessed at different time points (1-5 dpf). The results suggested that rapamycin resulted in a significant delay in the development of the embryos in a dose- and time-dependent manner, indicated by the alterations in body length (Fig. 4). Rapamycin treated embryos had larger yolks and shorter body sizes, and the effect of rapamycin on body size was more apparent at 10 and $20 \mu \mathrm{M}$ treatments compared with the $2 \mu \mathrm{M}$ treatment (Figs. 4 and 5). In particular, rapamycin treated embryos exhibited marked reduction in melanocyte distribution, with this observed to be dose-dependent (Fig. 5A and B).

\section{Discussion}

The present study, to the best of our knowledge, is the first characterization of the global transcriptional response of a zebrafish cell line (ZF4) when exposed to $100 \mathrm{nM}$ rapamycin. Rapamycin treatment in zebrafish was demonstrated to result in the coordinated modulation of genes that belonged to pathways important in cell growth, division and metabolism. Meta-analysis of heterogeneous mouse microarray datasets then enabled the identification of functional pathways similarly and significantly modulated in zebrafish and mice in response to rapamycin (Tables II and III). Thus, the current study represents an important step in an improved understanding of the functional conservation of mTOR inhibition by rapamycin among vertebrates.

Comparative KEGG pathway analysis identified that ribosome was the most significant pathway upregulated in both species when exposed to rapamycin, while proteasome complexes, oxidative phosphorylation, the cell cycle and certain metabolic pathways were commonly downregulated (Table II). A well-known effect of rapamycin on cellular metabolism is the inhibition of translation through the modulation of ribosomal subunits and/or eukaryotic translational factors (33). In the yeast model, rapamycin negatively affects both the steady state and polysome-associated mRNA levels of genes involved in RNA processing and ribosome biogenesis together with the proteasome (9). However, in reports regarding vertebrates, the effects of rapamycin on ribosomes have been attributed predominantly to alterations in the polysome-associated mRNA pool $(34,35)$. The comparative transcriptomics of the present study indicated that, in both zebrafish and mice, steady state mRNA levels of ribosomal subunits were upregulated coordinately. These results indicate that rapamycin may result in an increased accumulation of nuclear ribosomal subunit mRNAs in vertebrates. Although small in magnitude, this collective increase may actually be effectively large since ribosomal subunits are highly expressed.

The observed increase in nuclear ribosomal component expression may be the result of translation inhibition itself. With the stalling of translation or sequestering of mRNAs, a surplus of mRNA for the translational machinery may accumulate (36); however, this requires further investigation. Notably, a previous study indicated that translational inhibitors paradoxically induced the transcription of ribosomal architecture components and translational regulatory factors (37). The implications of an increase in the ribosomal mRNA pool in response to translational inhibition may be associated with the apoptotic and survival-associated functions of rapamycin treatment. It has been previously demonstrated that ribosomal subunit mRNAs may regulate apoptosis driven by $\mathrm{p} 53$, and thus have implications in cell survival and apoptosis (38).

Additionally, the current study demonstrated a coordinated but mild downregulation of a subset of the mitochondrial ribosomal proteins in rapamycin treated ZF4 cells and mouse cell lines, indicated by the significant enrichment of mitochondrion and mitochondrial part terms among downregulated GO-CC terms (data not shown, Benjamini <0.05). This result is in accord with previous studies reporting that rapamycin resulted in the downregulation of mitochondrial ribosomes (10). The proteasome was a significantly downregulated KEGG pathway, for both zebrafish and mice (Table II). Additionally, a previous study indicated that rapamycin was able to downregulate proteasome subunits at the transcriptional and translational levels in human E6-1 Jurkat T cells (34). Hence, by reducing proteasomal subunit expression, rapamycin may also act as a proteasomal inhibitor in zebrafish. 

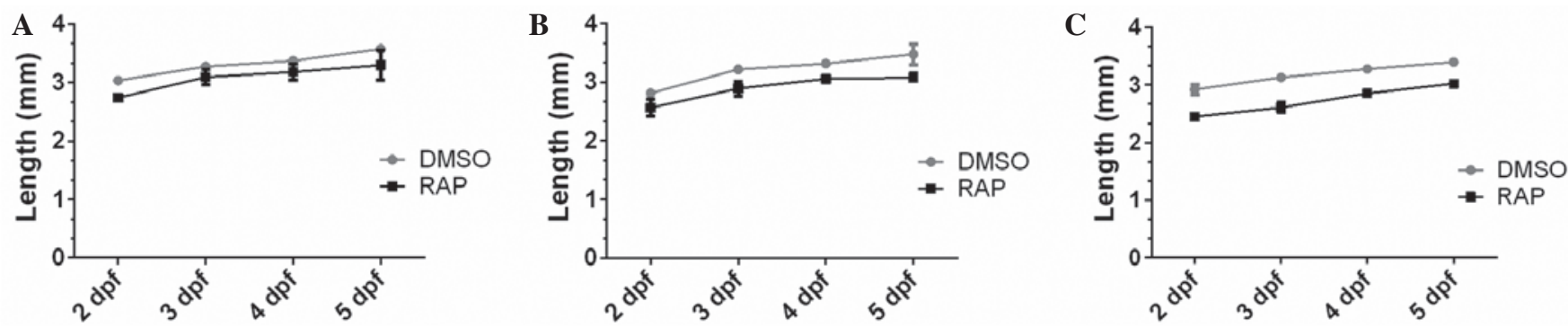

Figure 4. Time- and dose-dependent alterations in body size following treatment with (A) 2, (B) 10 and (C) $20 \mu \mathrm{M}$ rapamycin. The lengths of the fish were measured from the tip of the head to the end of notochord in $\mathrm{mm}$, and the mean plotted against the time of development. Data are presented as the mean \pm standard deviation. General linear model; $\mathrm{P}_{\text {dose }} \leq 0.001, \mathrm{P}_{\text {time }} \leq 0.001, \mathrm{P}_{\text {rapamycin }} \leq 0.001$. dpf, days post fertilization; DMSO, dimethyl sulfoxide; RAP, rapamycin.

\section{A \\ Rap \\ $1 \mathrm{dpf}$ \\ DMSO \\ $3 \mathrm{dpf}$ \\ Rap $3 \mathrm{dpf}$}
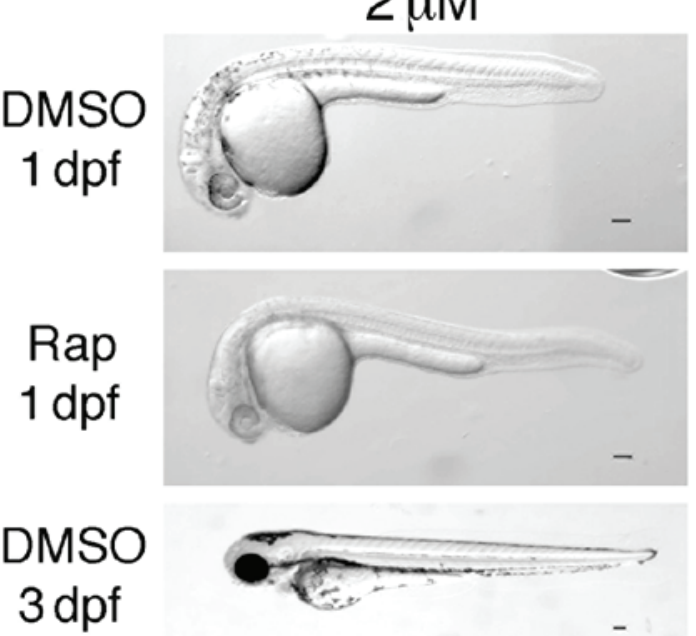

B

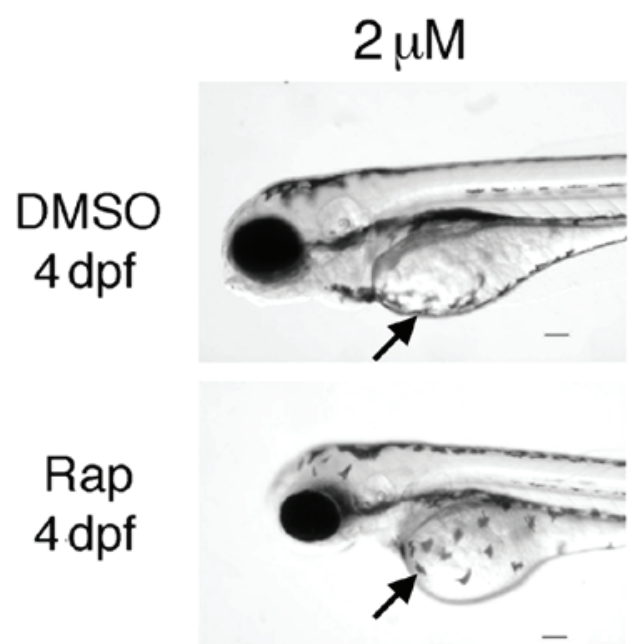

$10 \mu \mathrm{M}$

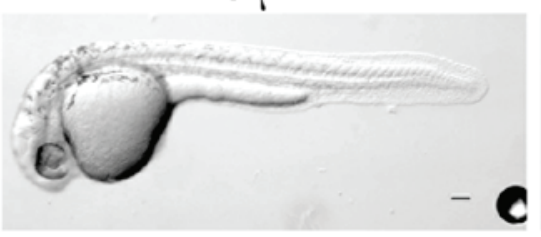

c
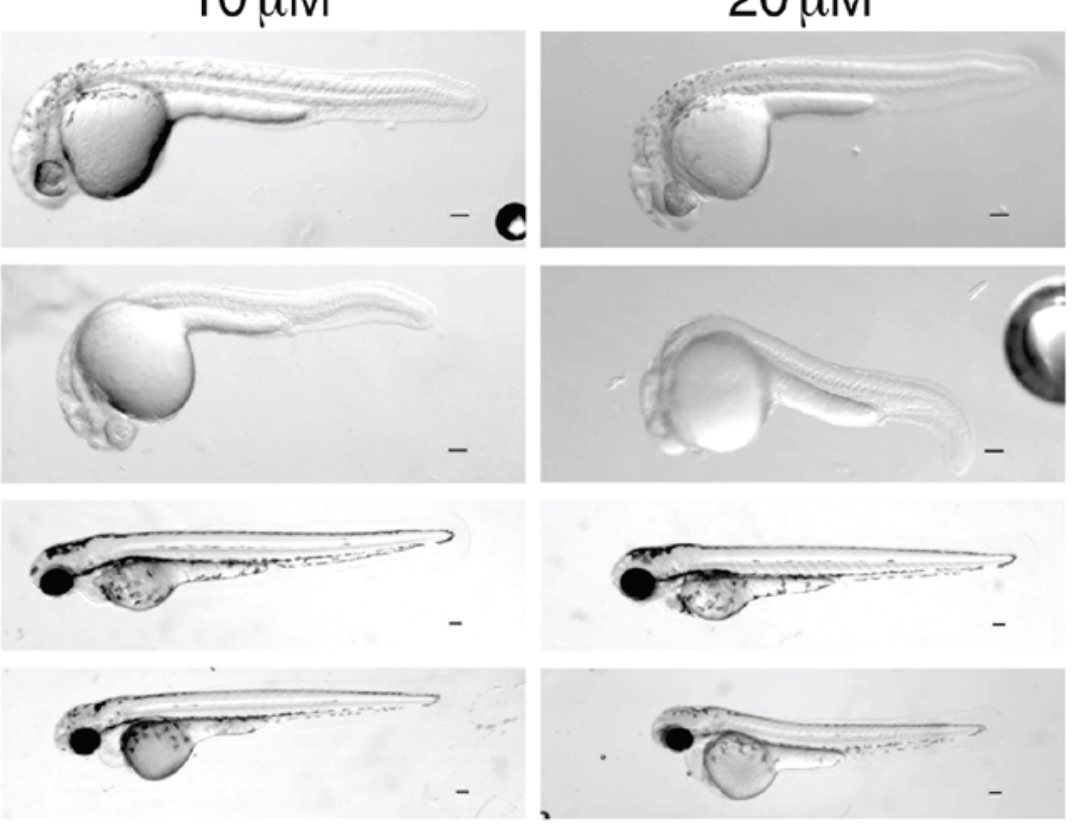

$20 \mu \mathrm{M}$
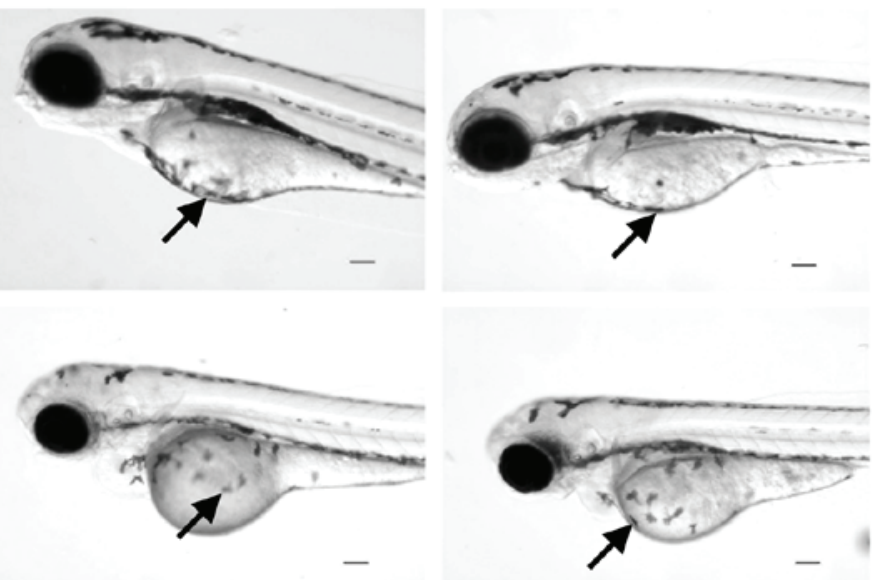

Figure 5. Effects of rapamycin treatment on the embryonic development of zebrafish. Shield stage zebrafish embryos were treated with 2, 10 and $20 \mu \mathrm{M}$ rapamycin or DMSO prior to collection at the indicated time points. (A) Rapamycin and DMSO treated embryos at 1 and 3 dpf. (B) Effect of rapamycin treatment on melanophore pigmentation (arrows) at 4 dpf. Scale bars $=100 \mu \mathrm{m}$. DMSO, dimethyl sulfoxide; dpf, days post fertilization; Rap, rapamycin.

Cellular analyses indicated that in ZF4 cells, rapamycin reduced the rate of basal apoptosis while having no significant effect on cell viability or proliferation. As previously indicated, rapamycin may induce or protect cells from apoptosis $(39,40)$. The current study suggests that rapamycin may have a protective effect against apoptosis in ZF4 cells that can potentially mask 
alterations in cell number and viability. The MTT test did not indicate a significant growth inhibition in $\mathrm{ZF} 4$ cells following rapamycin treatment, however, rapamycin was observed to significantly downregulate zebrafish and mouse KEGG and GO-BP pathways including the cell cycle (Tables II and IV).

Notably, the correlations among the three individual mouse dataset fold change values $\left(\log _{2}\right)$ were significantly high, ranging between 0.21 and 0.59 regardless of the mutations the cell lines carried (Tp53, Tsc1 and Tcs2 genes). This suggests that the meta-analysis used in the current study was able to capture a significant portion of the conserved aspects of rapamycin treatment in mice, which may then be extended to zebrafish, a non-mammalian vertebrate. A previous study showed that ZF4 cells exhibited a drastic cellular growth inhibition and a reduction in the expression of conserved E2F1 pathway-associated genes in response to serum starvation (41). Accordingly, the present study indicates that the ZF4 cell line is a valuable model for detecting conserved modulators of cell growth and division-associated genes and pathways.

Additionally, the present study provided a foundation for the identification and validation of novel genes affected by rapamycin treatment time-dependently using ZF4 fibroblast cells. The correlation between the log fold changes obtained from the independent RT-qPCR and microarray data in response to rapamycin was significantly high, however, it was only possible to confirm the significance of $55 \%$ of the genes by RT-qPCR. The discrepancies between the RT-qPCR and microarray results may result from the differences arising from the use of different cell passages in the experiments and/or potentially different probe and primer sequences/dynamics between the microarray and RT-qPCR methodologies (42). Upon validation by RT-qPCR, a significant modulation in response to rapamycin was observed in several pigmentation associated genes, with an increase in the expression of $d k k l b$ and $d d c$ and a reduction in pah expression (Fig. 2). In a previous study, the overexpression of DKK1 suppresses melanocyte growth and proliferation in human keratinocytes (43). DDC provides dopamine by conversion from dopa, which also can be used for melanin production, such as in butterfly wings (44). PAH supplies tyrosine for melanin production and contributes to melanogenesis (45), and PAH (Enu) mice have inactive TORC1 (46). The role of TOR signaling in melanin pigmentation has also been observed in Drosophila, such that increased TORC1 activity resulted in altered pigmentation (47).

Zebrafish embryos and adults have been shown to be sensitive to rapamycin. For instance, treatment with $400 \mathrm{nM}$ rapamycin resulted in a mild delay in embryonic development while arresting digestive tract development $72 \mathrm{hpf}$ following exposure (6). Similarly, $10 \mathrm{nmol} / 1$ rapamycin treatment phenocopied embryonic growth-associated protein morphants exhibiting developmental delays, vessel defects and cardiac failure (48). In addition, the growth of the adult caudal fin in zebrafish was negatively affected by treatment with rapamycin (49). The present in vivo study not only confirmed the growth limiting effects of rapamycin in zebrafish but also implicated a dose-dependent role for mTOR inhibition in pigmentation-associated processes. Indeed, a negative effect of rapamycin on melanocytes has been indicated in a Xenopus model (50), complementing the alterations observed in the present study regarding pigmentation upon rapamycin exposure in zebrafish (Fig. 5). However, the mechanisms underlying the effects of rapamycin on pigmentation should be explored at the transcript level in vivo.

In conclusion, the present study indicates that rapamycin modulates a wide range of transcripts in ZF4 cells, including nuclear and mitochondrial ribosomal machinery components and proteasomal subunits. These functional alterations were supported by KEGG and GO pathway meta-analysis of mouse microarray datasets of rapamycin exposure. This is the first report, to the best of our knowledge, of the conservation between the zebrafish and mouse functional pathway enrichment profiles in response to an mTOR inhibitor. Furthermore, the significantly affected genes produced high gene enrichment scores suggesting that a coordinated set of conserved events occurs in response to rapamycin in zebrafish and mice. Additionally, the results revealed that the response of zebrafish embryos to rapamycin exhibits similarities with those from other organisms, providing a foundation for comparative expression profiling studies of rapamycin treatment between zebrafish and mammals.

\section{Acknowledgements}

The authors wish to thank to Dr Carl J Neumann for enabling the in vivo zebrafish embryo studies to be conducted in his laboratory at the European Molecular Biology Laboratory (Heidelberg, Germany), Ms. Sabine Fischer for technical support and guidance, and Dr Gulcin Cakan Akdogan for help in the preparation of figures. The current study was funded by The Scientific and Technological Research Council of Turkey (grant nos. 103T038 and 105S365).

\section{References}

1. Corradetti MN and Guan KL: Upstream of the mammalian target of rapamycin: Do all roads pass through mTOR? Oncogene 25: 6347-6360, 2006.

2. Brown EJ, Albers MW, Shin TB, Ichikawa K, Keith CT, Lane WS and Schreiber SL: A mammalian protein targeted by G1-arresting rapamycin-receptor complex. Nature 369: 756-758, 1994

3. Akcakanat A, Singh G, Hung MC and Meric-Bernstam F: Rapamycin regulates the phosphorylation of rictor. Biochem Biophys Res Commun 362: 330-333, 2007.

4. Rao RD, Buckner JC and Sarkaria JN: Mammalian target of rapamycin (mTOR) inhibitors as anti-cancer agents. Curr Cancer Drug Targets 4: 621-635, 2004.

5. Loewith R, Jacinto E, Wullschleger S, Lorberg A, Crespo JL, Bonenfant D, Oppliger W, Jenoe P and Hall MN: Two TOR complexes, only one of which is rapamycin sensitive, have distinct roles in cell growth control. Mol Cell 10: 457-468, 2002.

6. Makky K, Tekiela J and Mayer AN: Target of rapamycin (TOR) signaling controls epithelial morphogenesis in the vertebrate intestine. Dev Biol 303: 501-513, 2007.

7. Ji J and Zheng PS: Activation of mTOR signaling pathway contributes to survival of cervical cancer cells. Gynecol Oncol 117: 103-108, 2010.

8. Jimenez RH, Lee JS, Francesconi M, Castellani G, Neretti N, Sanders JA, Sedivy J and Gruppuso PA: Regulation of gene expression in hepatic cells by the mammalian Target of Rapamycin (mTOR). PLoS One 5: e9084, 2010.

9. Preiss T, Baron-Benhamou J, Ansorge W and Hentze MW: Homodirectional changes in transcriptome composition and mRNA translation induced by rapamycin and heat shock. Nat Struct Biol 10: 1039-1047, 2003.

10. Cunningham JT, Rodgers JT, Arlow DH, Vazquez F, Mootha VK and Puigserver P: mTOR controls mitochondrial oxidative function through a YY1-PGC-1alpha transcriptional complex. Nature 450: 736-740, 2007. 
11. Duvel K, Yecies JL, Menon S, Raman P, Lipovsky AI, Souza AL, Triantafellow E, Ma Q, Gorski R, Cleaver S, et al: Activation of a metabolic gene regulatory network downstream of mTOR complex 1. Mol Cell 39: 171-183, 2010.

12. Totary-Jain H, Sanoudou D, Ben-Dov IZ, Dautriche CN, Guarnieri P, Marx SO, Tuschl T and Marks AR: Reprogramming of the microRNA transcriptome mediates resistance to rapamycin. J Biol Chem 288: 6034-6044, 2013.

13. Hentges KE, Sirry B, Gingeras AC, Sarbassov D, Sonenberg N, Sabatini D and Peterson AS: FRAP/mTOR is required for proliferation and patterning during embryonic development in the mouse. Proc Natl Acad Sci USA 98: 13796-13801, 2001.

14. Oldham S, Montagne J, Radimerski T, Thomas G and Hafen E: Genetic and biochemical characterization of dTOR, the Drosophila homolog of the target of rapamycin. Genes Dev 14: 2689-2694, 2000 .

15. Ganesan S, Moussavi Nik SH, Newman M and Lardelli M: Identification and expression analysis of the zebrafish orthologues of the mammalian MAP1LC3 gene family. Exp Cell Res 328: 228-237, 2014.

16. Sapp V, Gaffney L, EauClaire SF and Matthews RP: Fructose leads to hepatic steatosis in zebrafish that is reversed by mechanistic target of rapamycin (mTOR) inhibition. Hepatology 60: 1581-1592, 2014.

17. Burkhalter MD, Fralish GB, Premont RT, Caron MG and Philipp M: Grk5l controls heart development by limiting mTOR signaling during symmetry breaking. Cell Rep 4: 625-632, 2013.

18. Kim SH, Scott SA, Bennett MJ, Carson RP, Fessel J, Brown HA and Ess KC: Multi-organ abnormalities and mTORC1 activation in zebrafish model of multiple acyl-CoA dehydrogenase deficiency. PLoS Genet 9: e1003563, 2013.

19. Xu B, Lee KK, Zhang L and Gerton JL: Stimulation of mTORC1 with L-leucine rescues defects associated with Roberts syndrome. PLoS Genet 9: e1003857, 2013.

20. Li Y, Huang W, Huang S, Du J and Huang C: Screening of anti-cancer agent using zebrafish: Comparison with the MTT assay. Biochem Biophys Res Commun 422: 85-90, 2012.

21. Driever W and Rangini Z: Characterization of a cell line derived from zebrafish (Brachydanio rerio) embryos. In Vitro Cell Dev Biol Anim 29A: 749-754, 1993

22. Krishan A: Rapid flow cytofluorometric analysis of mammalian cell cycle by propidium iodide staining. J Cell Biol 66: 188-193, 1975.

23. Gautier L, Cope L, Bolstad BM and Irizarry RA: Affy-analysis of Affymetrix GeneChip data at the probe level. Bioinformatics 20: 307-315, 2004

24. Bolstad BM, Collin F, Brettschneider J, Simpson K, Cope L, Irizarry RA and Speed TP: Quality assessment of affymetrix GeneChip data. In: Bioinformatics and Computational Biology Solutions Using R and Bioconductor. Gentleman R, Carey V, Huber W, Irizarry R and Dudoit S (eds.). Springer, New York, pp 33-47, 2005.

25. Huang da W, Sherman BT and Lempicki RA: Systematic and integrative analysis of large gene lists using DAVID bioinformatics resources. Nat Protoc 4: 44-57, 2009.

26. Huang da W, Sherman BT and Lempicki RA: Bioinformatics enrichment tools: Paths toward the comprehensive functional analysis of large gene lists. Nucleic Acids Res 37: 1-13, 2009.

27. Wang X, Kang DD, Shen K, Song C, Lu S, Chang LC, Liao SG, Huo Z, Tang S, Ding Y, et al: An R package suite for microarray meta-analysis in quality control, differentially expressed gene analysis and pathway enrichment detection. Bioinformatics 28 : 2534-2536, 2012

28. McCurley AT and Callard GV: Characterization of housekeeping genes in zebrafish: Male-female differences and effects of tissue type, developmental stage and chemical treatment. BMC Mol Biol 9: 102, 2008.

29. Pfaffl MW: A new mathematical model for relative quantification in real-time RT-PCR. Nucleic Acids Res 29: e45, 2001.

30. Fay MP: Confidence intervals that match Fisher's exact or Blaker's exact tests. Biostatistics 11: 373-374, 2010.

31. Yildiz G, Arslan-Ergul A, Bagislar S, Konu O, Yuzugullu H, Gursoy-Yuzugullu O, Ozturk N, Ozen C, Ozdag H, Erdal E, et al: Genome-wide transcriptional reorganization associated with senescence-to-immortality switch during human hepatocellular carcinogenesis. PLoS One 8: e64016, 2013.
32. Wright GW and Simon RM: A random variance model for detection of differential gene expression in small microarray experiments. Bioinformatics 19: 2448-2455, 2003.

33. Terada N, Patel HR, Takase K, Kohno K, Nairn AC and Gelfand EW: Rapamycin selectively inhibits translation of mRNAs encoding elongation factors and ribosomal proteins. Proc Natl Acad Sci USA 91: 11477-11481, 1994.

34. Grolleau A, Bowman J, Pradet-Balade B, Puravs E, Hanash S, Garcia-Sanz JA and Beretta L: Global and specific translational control by rapamycin in $\mathrm{T}$ cells uncovered by microarrays and proteomics. J Biol Chem 277: 22175-22184, 2002.

35. Parent R, Kolippakkam D, Booth G and Beretta L: Mammalian target of rapamycin activation impairs hepatocytic differentiation and targets genes moderating lipid homeostasis and hepatocellular growth. Cancer Res 67: 4337-4345, 2007.

36. Moore MJ: From birth to death: The complex lives of eukaryotic mRNAs. Science 309: 1514-1518, 2005.

37. Boshoff HI, Myers TG, Copp BR, McNeil MR, Wilson MA and Barry CE III: The transcriptional responses of Mycobacterium tuberculosis to inhibitors of metabolism: Novel insights into drug mechanisms of action. J Biol Chem 279: 40174-40184, 2004.

38. Bhat KP, Itahana K, Jin A and Zhang Y: Essential role of ribosomal protein L11 in mediating growth inhibition-induced p53 activation. EMBO J 23: 2402-2412, 2004

39. Ravikumar B, BergerZ, Vacher C, O'Kane CJ and Rubinsztein DC Rapamycin pre-treatment protects against apoptosis. Hum Mo Genet 15: 1209-1216, 2006.

40. Tirado OM, Mateo-Lozano S and Notario V: Rapamycin induces apoptosis of JN-DSRCT-1 cells by increasing the Bax: $\mathrm{Bcl}-\mathrm{xL}$ ratio through concurrent mechanisms dependent and independent of its mTOR inhibitory activity. Oncogene 24: 3348-3357, 2005

41. Sucularli C, Senturk S, Ozturk M and Konu O: Dose- and time-dependent expression patterns of zebrafish orthologs of selected E2F target genes in response to serum starvation/replenishment. Mol Biol Rep 38: 4111-4123, 2011.

42. Dallas PB, Gottardo NG, Firth MJ, Beesley AH, Hoffmann K, Terry PA, Freitas JR, Boag JM, Cummings AJ and Kees UR: Gene expression levels assessed by oligonucleotide microarray analysis and quantitative real-time RT-PCR-how well do they correlate? BMC Genomics 6: 59, 2005.

43. Yamaguchi Y, Passeron T, Hoashi T, Watabe H, Rouzaud F, Yasumoto K, Hara T, Tohyama C, Katayama I, Miki T and Hearing VJ: Dickkopf 1 (DKK1) regulates skin pigmentation and thickness by affecting Wnt/beta-catenin signaling in keratinocytes. FASEB J 22: 1009-1020, 2008.

44. Koch PB, Keys DN, Rocheleau T, Aronstein K, Blackburn M, Carroll SB and ffrench-Constant RH: Regulation of dopa decarboxylase expression during colour pattern formation in wild-type and melanic tiger swallowtail butterflies. Development 125: 2303-2313, 1998.

45. Schallreuter KU and Wood JM: The importance of L-phenylalanine transport and its autocrine turnover to L-tyrosine for melanogenesis in human epidermal melanocytes. Biochem Biophys Res Commun 262: 423-428, 1999.

46. Kwak SS, Suk J, Choi JH, Yang S, Kim JW, Sohn S, Chung JH, Hong YH, Lee DH, Ahn JK, et al: Autophagy induction by tetrahydrobiopterin deficiency. Autophagy 7: 1323-1334, 2011.

47. Zitserman D, Gupta S, Kruger WD, Karbowniczek M and Roegiers F: The TSC $1 / 2$ complex controls Drosophila pigmentation through TORC1-dependent regulation of catecholamine biosynthesis. PLoS One 7: e48720, 2012.

48. Wenzlau JM, Garl PJ, Simpson P, Stenmark KR, West J, Artinger KB, Nemenoff RA and Weiser-Evans MC: Embryonic growth-associated protein is one subunit of a novel N-terminal acetyltransferase complex essential for embryonic vascular development. Circ Res 98: 846-855, 2006.

49. Goldsmith MI, Iovine MK, O'Reilly-Pol T and Johnson SL: A developmental transition in growth control during zebrafish caudal fin development. Dev Biol 296: 450-457, 2006.

50. Moriyama Y, Ohata Y, Mori S, Matsukawa S, Michiue T, Asashima $M$ and Kuroda $H$ : Rapamycin treatment causes developmental delay, pigmentation defects and gastrointestinal malformation on Xenopus embryogenesis. Biochem Biophys Res Commun 404: 974-978, 2011. 